\begin{abstract}
The purpose of this study was to compere the onset and severity of ingivitis in children with Down androme, when compared to a healthy control group of children. The subjects included $\mathbf{4 1}$ children with Down cyndrome ages two to 14 years (mean 9ge: 7.6 yeara) and 112 age-matched healthy controls. We assessed the gingival health of all subjects using the gingival inflammation (M-PMA) index and periodontal probing depth (PD). Children were divided into three age categories: $<5$ years (Al), 5 to $<10$ years (AI), and 10 to $<17$ years (AIII).

Supregingival plaque was measured using the Oral Hygiene Index (OHI) and the subjects were screened with the BANA test (Perioscan-Oral-B). Measurement of the M-PMA index in the healthy children showed an age-related increase $(F=10.369, p<0.001)$, and the $M-P M A$ index at the younger age group $<5$ year CAD was significantly lower than that for the other two age groups All or All $(p<0.005, p<0.001)$. In contrast, the M-PMA index values at $A$ and All in the subjects with Down syndrome were significantly higher than those for healthy children $(p<0.001, p<0.001)$. Both groups had an age-related increase in $P D(F=3.388, p<0.05 \& F=10.806$, $p<0.001$ ), and $P D$ at All was significantly higher than that at $A l$ in both groups $(p<0.04, p<0.001)$. The children with Down syndrome showed an age-related increase in the BANA test score $(F=3.452, p<0.05$ ), and the BANA test score at All was significantly higher than that at Al $(p<0.02)$. The BANA test score in the healthy children was not age-related but was significantly higher than that in the children with Down syndrome $(p<0.02, p<0.05)$.
\end{abstract}

KAY WORDSF BANA test, children, Down syndrome, gingivitis, pathogenesis. periodontal disease, progression

\title{
A Comparison of the Gingival Health of Children with Down Syndrome to Healthy Children Residing in an Institution
}

\author{
Takanobu Morinushi'"; Dennis E. Lopatin²; Rie Nakao'; Sachiko Kinjyo' \\ ${ }^{2}$ Dept. of Pediatric Dentistry, Kagoshima University Dental School, Kagoshima, Japan; ${ }^{2}$ Dept. of Biologic \\ and Materials Sciences, School of Dentistry, University of Michigan, Ann Arbor, Michigan, USA; \\ *Corresponding author: mori@dentb.hal.kagoshim-u.ac.jp \\ Spec. Care Dentist 26(1): 13-19, 2006
}

\section{Introduction}

Down syndrome is a genetic disease resulting from trisomy of the 21 st chromosome. The overall prevalence of Down syndrome in Japan is 5.82 per 10,000 live births. ${ }^{1}$ It has been reported that periodontal disease occurs, with high prevalence, in preschool children with Down syndrome and that its features are similar to those of severe juvenile periodontal disease..$^{2.4}$ It has been suggested that the early onset and severity of periodontal disease is related to immunological dysfunctions, including neutrotaxis, dysfunction of T-cells, deficient phagocytosis and increased prostaglandin E2 levels in gingival sulcus fluid. ${ }^{5-10}$ Cichon et al ${ }^{11}$ reported that changes in the immunological response due to alterations of the subgingival flora were one of the causes of periodontal disease in persons with Down syndrome. Periodontopathic bacteria in persons with Down syndrome have also been investigated. ${ }^{12.13}$ These studies have suggested that periodontopathogens in preschool-age children with Down syndrome become established in early childhood, usually with Actinobacillus actinomycetemcomitans in gingivitis and Porphyromonas gingivalis in periodontitis, with both the early onset of periodontal disease and the tendency for the disease to be severe. However, few studies have examined the differences in gingivitis caused by these two species of bacteria in children with and without Down syndrome. ${ }^{14-16}$ Although reports have described various factors that might contribute to periodontal disease in children with Down syndrome, no adequate explanation for the early onset of gingivitis and the tendency for severe periodontal disease in these children has been reported.

While several studies ${ }^{17,18}$ have shown that periodontal treatment did not inhibit the progression of periodontal disease in subjects with Down syndrome, the effectiveness of frequent preventive care and periodontal treatment in these subjects has only recently been reported. ${ }^{19,20}$ These reports suggest that an immunological problem is not a factor that makes prevention and care of periodontal disease in patients with Down syndrome difficult. To further elucidate the characteristics of periodontal disease in Down syndrome, it is important to do standard clinical evaluations and also examine the exposure to periodontopathogens in children with Down syndrome during childhood and adolescence and compare it with that of healthy children. This study evaluated the generally accepted reports of early onset of gingivitis and examined the progression of periodontal disease in subjects with Down syndrome undergoing nonspecific periodic preventive care, compared with that of healthy children residing in an institution. 
Table 1. Demographics of study subjects

Age Group Categories Down Syndrome Patients Healthy Children

Male Female Total Average Age Male Female Total Average Age

$\begin{array}{lllllllllll}<5 \mathrm{yr} . & \mathrm{AI}-\mathrm{G} & 9 & 4 & 13 & 3.3 \mathrm{yr} & 9 & 4 & 13 & 2.7 \mathrm{yr} .\end{array}$

$\begin{array}{ccccccccccc}5 \text { to }<10 \mathrm{yr} . & \text { AII-G } & 9 & 6 & 15 & 7.9 \mathrm{yr} & 23 & 14 & 37 & 6.9 \mathrm{yr} . \\ >=10 \mathrm{yr} . & \text { AIII-G } & 9 & 4 & 13 & 11.6 \mathrm{yr} & 35 & 27 & 62 & 11.9 \mathrm{yr} . \\ & & & & & & & & & & \\ \text { Total } & & 27 & 14 & 41 & 7.6 \mathrm{yr} & 37 & 45 & 112 & 9.2 \mathrm{yr} .\end{array}$

\section{Materials and Methods}

\section{Subjects}

This study was carried out in accordance with the Helsinki Declaration of 1975, as revised in 1983. Informed consent was obtained from the subjects, their parents and the persons in charge at the institutions.

The group with Down syndrome consisted of 41 children ( 27 boys and 14 girls) ages two to 14 years old (average age: 7.6 years), who were patients of the Department of Pediatric Dentistry in the hospital affiliated with the University School of Dentistry. The control group consisted of 112 healthy children (67 boys and 45 girls) ages two to 14 years old (average age: 9.2 years) living in institutions for homeless or neglected children (Table 1). The criteria for inclusion were as follows: 1) no antibiotic treatment for at least three months before the sampling; 2) informed consent from subjects in both the group with Down syndrome and the control group; and 3) establishment of a friendly relationship between the examiner and subjects before sampling. The subjects were divided into three age categories: $<5$ years $(\mathrm{AI}), 5$ to $<10$ years (AII) and 10 to $<17$ years (AIII).
The subjects with Down syndrome received an oral examination and preventive care four times a year. The preventive care treatments included professional tooth cleaning, various combinations of scaling, counseling of the caregiver and fluoride treatment for caries and periodontal disease therapy. The healthy children received only an oral examination twice a year without fluoride treatment and did not receive specific instructions about prevention or other treatment.

\section{Clinical Examinations}

One examiner performed all of the clinical examinations.

Gingival condition-The degree of gingival inflammation was scored using the Modified Total PMA (M-PMA) Index based on the PMA Index proposed by Massler. ${ }^{21}$ The presence or absence of gingivitis was noted in each of the following three areas in the buccal or labial gingiva: the gingival papillae $(P)$, the gingival margin (M) and the attached gingiva (A). Each of these areas was scored according to the presence or absence of inflammation as 1 or 0 , respectively. The M-PMA index of each subject was defined as M-PMA index=(summation of PMA indices)/ (number of examined areas) $\times 100(\%)$.
Extent of supragingival plaque-The extent of supragingival plaque (PI) was evaluated by stratifying plaque in the subject's teeth into four grades based on the Oral Hygiene Index proposed by Greene \& Vermillion. ${ }^{22}$

Microbiological examinationsSubgingival plaque samples were analyzed for the presence of anaerobic periodontal pathogens, Porphyromonas gingivalis, Treponema denticola and Bacteroides forsythus, using the BANA (benzoyl-DL-arginine-naphthylamide) test (Perioscan ${ }^{\circledast}$, Oral-B Laboratories Inc., Redwood City, CA $)^{23-25}$. After the removal of supragingival plaque, subgingival plaque samples were taken from mesiobuccal surfaces of four first molars or deciduous second molars, and four central incisors or deciduous central incisors using a No. 6 dental explorer, and were then wiped onto BANAimpregnated lower strips at the bottoms of the BANA reagent cards. If these teeth were not present, plaque samples from adjacent teeth were used. The reaction was immediately activated by water applied to the upper strip containing fast black dye. The lower strip was folded onto the upper strip, and both strips were held in place with a metallic clip. The card was placed in an incubator at 
Table 2. Relationship Between Age and Gingivitis

Total-PMA Score

Age Categories Down Syndrome Group Healthy children group Significance*

Mean \pm SD Mean \pm SD

\begin{tabular}{|c|c|c|c|c|c|c|}
\hline AI-G (<5yr. $)$ & 0.312 & \pm 0.179 & $>$ & 0.052 & $\pm 0 . \overline{06}\rceil$ & $\mathrm{p}<0.001$ \\
\hline \multirow[t]{2}{*}{ AII-G $(5$ to $<10 y r)}$. & 0.309 & \pm 0.189 & & 0.215 & $\pm 0.14 \underline{9}$ & NS \\
\hline & & & & \multicolumn{3}{|c|}{$\mathrm{P}<0.0001$} \\
\hline \multirow[t]{2}{*}{ AIII-G (>=10yr.) } & 0.422 & \pm 0.122 & $>$ & 0.244 & \pm 0.140 & $\mathrm{p}<0.001$ \\
\hline & $F=1.961$ & - & \multicolumn{2}{|c|}{$F=10.369$} & 0001 & \\
\hline
\end{tabular}

*Differences between Down and control subjects were assessed by Mann-Whitney U test.

Differences between age categories were assessed by ANOVA.

$55^{\circ}$ for 15 minutes. Results were recorded as "strong, dark-blue spots" (score = 3), "weak, light-blue spots" (score $=2$ ) or "no color change" (score = 1). The BANA score of each subject was defined as mean values of BANA scores of the eight examined teeth.

Probing depth-Probing depth (PD) was measured to the nearest millimeter with a round-ended probe of $0.4 \mathrm{~mm}$ in diameter at the same eight sites as those used those for the BANA test.

\section{Analysis}

All clinical index values were mean values, obtained by dividing the sum total of all individual measurements by the number of measurements. The Mann-Whitney U-test was used to assess mean values between the two groups. Distributions among the three age categories were assessed by ANOVA. Correlation was estimated by using
Spearman's correlation coefficient by ranks. A $p$-value $<0.05$ was considered statistically significant.

\section{Results}

Several significant correlations between clinical indices were identified when individuals within specific age categories in the control group were examined. For example, for children in the age group 5 to $<10$ years (AII), there was a significant correlation between M-PMA and PD $(z=2.808, p<0.005)$, and significant correlations were found between M-PMA and PI $(z=3.787$, $\mathrm{p}<0.001)$ and between M-PMA and PD $(z=3.004, p<0.001)$ in the control subjects.

\section{Relationship between Age and Gingivitis}

No significant change in M-PMA index was found in any of the age groups in the children with Down syndrome.
[Table 2] By comparison, in the healthy children, the M-PMA index increased in the age group AIII compared to that at $\mathrm{AI}(\mathrm{F}=10.369, \mathrm{p}<0.001)$, and the M-PMA index for children aged $<5$ years (AI) was significantly lower than that for AII or AlII $(p<0.001, p<0.001)$. When the two sample groups of children were compared for each category, M-PMA values for $\mathrm{AI}$ and AIII, the children with Down syndrome had significantly higher readings $(p<0.001, p<0.001)$ than those for the healthy children.

\section{Probing Depth}

The children with Down syndrome were found to have a significant increase in probing depth with increasing age $(\mathrm{F}=3.388, \mathrm{p}<0.05)$. The probing depth for the AIII age group was significantly greater than that for $\mathrm{AI}(\mathrm{p}<0.01)$

[Table 3]. The healthy children also had an age-related increase in probing depth $(F=10.806, p<0.0001)$. Probing depth for 
children aged $<5$ years was significantly less than that for the other two age groups $(\mathrm{p}<0.003, \mathrm{p}<0.0001)$. When similar age categories of children with Down syndrome were compared to the control group, no significant differences in probing depth were found.

\section{Relationships with Results of the BANA Test}

When plaque samples from the children with Down syndrome were examined, a significant age-related increase in BANA score was found $(F=3.452, p<0.05)$. Similarly, BANA scores for the oldest children were significantly higher than for the youngest $(p<0.02)$. The BANA scores of the healthy children, however, showed no significant relationship with age. When the BANA scores of both sample groups were compared for each age category, the children in the control group had significantly higher scores for age group
$\mathrm{AI}$ and AII than did the children with Down syndrome $(\mathrm{p}<0.02, \mathrm{p}<0.05)$ (Table 4).

\section{Plaque Index}

No significant age-related changes were noted in the plaque index, either in the group of children with Down syndrome or the control group.

\section{Discussion}

It has been reported that children with Down syndrome develop periodontal disease early in childhood at a comparatively high rate, with clinical findings similar to those of severe juvenile periodontal disease..$^{2-4}$ These findings have been attributed to immunological deficits. ${ }^{6-10,26}$ The microbiota associated with periodontal disease in children with Down syndrome have also been reported, ${ }^{12-13}$ suggesting that periodontopathogens in preschool-age children with Down syndrome become established in early childhood. It has been reported that Actinobacillus actinomycetemcomitans in gingivitis and Porphyromonas gingivalis in periodontitis are associated with early onset and a tendency for the disease to be severe. ${ }^{12,13}$ Because of these factors, the occurrence of periodontal disease in subjects with Down syndrome might be unavoidable, and it has been thought that the progress of the disease cannot be prevented. However, some recent reports ${ }^{9,27-28}$ have suggested the effectiveness of preventive care in suppressing the onset and progression of periodontal disease in this population. Shapira et $\mathrm{al}^{27}$ reported that preventing periodontal disease was possible by instituting preventive dental health programs, and Randell et $\mathrm{al}^{28}$ suggested that prevention should be possible by providing better dental health services for children with Down syndrome. Sakellari et al ${ }^{19}$ described

Table 3. Relationship Between Probing Depth and Age

\section{Age Categories Down Syndrome Group Healthy Children group Significance*}

Mean \pm SD Mean \pm SD

AI-G (<5yr)

2.215

AII-G $(5$ to $<10 y r)$.

2.588

AIII-G ( > =10yr.)

2.595

$F=3.388$

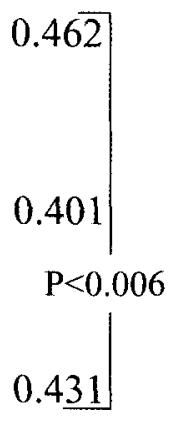

$\mathrm{p}<0.05$
2.202

2.744

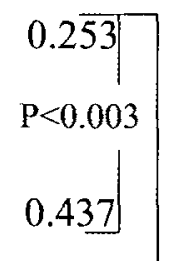

NS

$\mathrm{P}<0.0001$

2.830

$0 . \underline{475}$

NS

*Differences between Down and control subjects were assessed by Mann-Whitney U test. Differences between age categories were assessed by ANOVA. 
Table 4. Relationship Between BANA Score and Age

BANA Score

Age Categories Down Syndrome Group Healthy children Group Significance*

Mean \pm SD $\quad$ Mean \pm SD

AI-G (<5yr.)

1.471

AII-G $(5$ to $<10 y r$.

AIII-G (>=10yr.)
$0.4 \overline{7}<$

$\left.0.388\right|_{\mathrm{P}<0.02}<$
1.904

2.052

$0.27 \underline{6}$

0.30

$\mathrm{p}<0.02$

$\mathrm{F}=3.452 \mathrm{p}<0.05$

$\mathrm{F}=0.904$

*Differences between Down and control subjects were assessed by Mann-Whitney U test.

Differences between age categories were assessed by ANOVA.

the positive effects of closely monitoring the oral hygiene habits and frequent professional dental cleaning of five subjects with Down syndrome ages 2637 years. Lopez et $\mathrm{al}^{29}$ reported that the overall characteristics of the periodontal and gingival health status in children with Down syndrome who received preventive care were not markedly different from those in healthy children. The results of our study agree with those reported by Lopez et al, ${ }^{29}$ in that we found no marked differences between the two groups in probing depth and plaque index.

Our study focused on the difference between the M-PMA index in children with Down syndrome and that of healthy children in three age categories. In the healthy children, the M-PMA index at the youngest age was significantly lower than that for age groups AII or AIII. In the children with Down syndrome, however, no significant difference in the
M-PMA index was found in any of the age groups (AI to AIII). Furthermore, when the groups were compared at each age category, M-PMA values for age groups AI and AIII in the children with Down syndrome were significantly greater than those for the healthy children. Our results help to confirm the theories of early onset of periodontal disease in children with Down syndrome and also confirm that there is a greater severity of gingivitis in children with Down syndrome when compared to a control group of healthy children.

Modeer et $\mathrm{al}^{30}$ reported that alveolar bone loss, as a sign of periodontitis, was more frequently observed in children with Down syndrome ages 10 to 19 years than in healthy children, and that the symptoms tended to appear before age 11. If we assume $4 \mathrm{~mm}$ or more of probing depth to be periodontitis, our study does not support these conclusions. Barr-Agholme et $\mathrm{al}^{31}$ and
Nakagawa et al $^{32}$ reported that the occurrence of periodontitis is related to their adolescence. The mean age of the subjects in the Modeer et al study ${ }^{30}$ was 15.5 years, whereas the mean age of the subjects in our study was 7.6 years. In addition, our study did not find $4 \mathrm{~mm}$ or more of probing depth in any of the subjects. In another study, subjects older than age 15 showed no significant increase in M-PMA index compared to subjects younger than age 15 .

Several reports have described the relationship between childhood periodontal disease and the flora in dental plaque. Nakagawa et al ${ }^{33}$ described the association of Porphyromonas gingivalis and Actinobacillus actinomycetemcomitans with adolescent gingivitis and adult periodontitis. Morinushi et al ${ }^{16}$ reported that these microorganisms were present in infancy and were associated with gingivitis. Abraham et $\mathrm{al}^{34}$ acknowledged the 
relationship between these two types of bacteria and periodontitis. A review by Robert et $\mathrm{al}^{35}$ described associations of destructive periodontitis in children affected with Actinobacillus actinomycetemcomitans, Prevotella intermedia and Spirochetes. Barr-Agholme et $\mathrm{al}^{36}$ reported higher levels of Actinobacillus. actinomycetemcomitans and Capnocytophaga in subgingival plaque in children with Down syndrome compared to the level in the healthy control group, while Santos et al $^{37}$ confirmed the existence of high level of A. actinomycetemcomitans in children with Down syndrome. Morinushi et al ${ }^{12}$ examined the relationship between gingivitis in children with Down syndrome younger than age five and the microbiota associated with periodontal disease. They reported that adolescent gingivitis in their subjects was related to $P$. gingivalis, A. actinomycetemcomitans, Selenomonas and Srep. mitis. Amano et $\mathrm{al}^{13}$ reported that $B$. forsythus, T. denticola, P. nigrescens and Campylobacter rectus were detected by polymerase chain reaction in all age groups of children with Down syndrome and that the frequency of detection of $P$. gingivalis was significantly higher in these children after age five. These results suggest that $P$. gingivalis and $A$. actinomycetemcomitans are involved in periodontal disease in children, and that the early onset of and tendency for gingival inflammation to be severe in children with Down syndrome are related to the early establishment of these periodontopathogens.

The BANA test used in this study is a presumptive test for the periodontal pathogens $T$. denticola, $P$. gingivalis and $B$. forsythus. Studies using "normal" subjects have demonstrated that Porphyromonas gingivalis, Treponema denticola and Bacteroides forsythus have been frequently associated with adult forms (periodontitis) of periodontal disease. ${ }^{10,13,14,15,25}$

Furthermore, these BANA test-positive bacterial species may also be involved in periodontal disease seen in subjects with Down syndrome, and BANA test scores have been significantly associated with probing depths in adult subjects with Down syndrome. ${ }^{16}$ All subjects in the previous studies were older than age 16 , and all had periodontitis. In our study, children with Down syndrome who had a mean age of 7.6 years, did not have a significant correlation between BANA test scores and other indices (M-PMA, PD). The mean BANA test score in each age category of both groups of children was low (under 2). Unfortunately, Actinobacillus actinomycetemcomitans, which is thought to be closely related to gingivitis, could not be found in our present study.

In summary, this study showed that an early onset of gingivitis did occur in the children with Down syndrome and was more severe than in the healthy children. Probing depths in both groups increased with age. Despite the lower BANA test scores in all age groups of subjects with Down syndrome when compared with those of the healthy children, only the BANA test score for age group AIII was significantly higher than that of children for AI. The M-PMA index score at AIII in the children with Down syndrome was the highest of all the age categories. We speculate, therefore, that children with Down syndrome show a greater gingival inflammatory reaction to the periodontopathogens than healthy children, but that the progression of periodontal disease in subjects with Down syndrome can be controlled by routine preventive care, at least for those younger than age 15 years.

\section{Acknowledgments}

The authors wish to thank Dr. Walter Loesche for the generous gift of BANA test kits.

\section{References}

1. Hoshi $\mathrm{N}$, Hattori R, Hanatani K, Okuyama $\mathrm{K}$, Yamada H, Kishida T, et al. Recent trends in the prevalence of Down's syndrome in Japan, 1980-1997. Am J Med Genet 1999; 84:340-5.

2. Reuland-Bosma W, van-Dijk J, van-derWeele L. Experimental gingivitis around deciduous teeth in children with Down's syndrome. J Clin Periodontol 1986; 13:294-300
3. Barnett ML, Barr M, Friedman D, Sonnenberg EM. The prevalence of periodontitis and dental caries in a Down's syndrome population. J Periodontol 1986; 57:288-93

4. Reuland-Bosma W, van-Dijk J. Periodontal disease in Down's syndrome: a review. J Clin Periodontol 1986; 13:64-73.

5. Reuland-Bosma W, van-den-Barselaar MT, van-de-Gevel JS, Leijh-PC. Nonspecific and specific immune responses in a child with Down's syndrome and her sibling. A case report. J Periodontol 1988; 59:249-53.

6. Izumi Y, Sugiyama S, Shinozuka $O$, Yamazaki T, Ohyama T, Ishikawa I. Defective neutrophil chemotaxis in Down's syndrome patients and its relationship to periodontal destruction. J Periodontol 1989; 60:238-42.

7. Sreedevi H, Munshi SK. Neutrophil chemotaxis in Down's syndrome and normal children to Actinobacillus actinomycetemcomitans. J Clin Pediatr Dent 1988; 22:141-6.

8. Nicolo M, Bucci P, Mignogna MD, Amato M. Periodontitis associated with systemic diseases with qualitative deficiency of phagocyte function. II. Down's syndrome. Minerva Stomatol 1989; 38:905-9.

9. Barr-Agholme M, Krekmanova L, YucelLindberg T, Shinoda K, Modeer T. Prostaglandin E2 level in gingival crevicular fluid from patients with Down's syndrome. Acta Odontol Scand 1997; 55:101-5.

10. Barr-Agholme $M$, Dahllof $G$, Modeer $T$, Engstrom PE, Engstrom GN. Periodontal conditions and salivary immunoglobulins in individuals with Down's syndrome. J Periodontol 1998; 69:1119-23.

11. Cichon P, Crawford L, Grimm WD. Earlyonset periodontitis associated with Down's syndrome-clinical interventional study. Ann Periodontol 1998; 3:370-80.

12. Morinushi $\mathrm{T}$, Lopatin DE, Van Poperin $\mathrm{N}$. The relationship between gingivitis and the serum antibodies to the microbiota -associated with periodontal disease in children with Down's syndrome. J Periodontal 1997; 68:626-31.

13. Amano A, Kishima T, Kimura S, Takiguchi M, Ooshima T, Hamada S et al. Periodontopathic bacteria in children with Down's syndrome. J Periodontol 2000; 71:249-55. 
14. Nakagawa S, Tonogi N, Kubo S, Machida Y, Okuda K, Takazoe I. Subgingival microflora in children of early childhood, school age and circumpuberty. The proportion a frequency of Gram-negative bacteria in periodontally healthy and gingival groups. Shoni Shikagaku Zasshi 1991; 29:72-85.

15. Genco RJ. Current view of risk factors for periodontal diseases. J Periodontol 1996; 67(10 suppl):1041-9.

16. Morinushi T, Lopatin DE, Van Poperin N, Ueda Y. The relationship between gingivitis and colonization by Porphyromonas gingivalis and Actinobacillus actinomycetemcomitans in children. J Periodontol 2000; 71(3):403-409.

17. Saxen L, Aula S. Periodontal bone loss in patients with Down's syndrome: a follow-up study. J Periodontol 1982; 53(3):158-162.

18. Hanookai D, Nowzari $H$, Contreras A, Morrison JL, Slots J. Herpesviruses and periodontopathic bacteria in Trisomy 21 periodontitis. J Periodontol 2000; 71(3):376-384.

19. Sakellari D, Belibasakis G, Chadjipadelis T, Arapostathis K, Konstantinidis A. Supragingival and subgingival microbiota of adult patients with Down's syndrome. Changes after periodontal treatment. Oral Microbiol Immunol 2001; 16(6):376-382.

20. Randell DM, Harth S, Seow WK. Preventive dental health practices of non-institutionalized Down's syndrome children: a controlled study. J Clin Pediatr Dent 1992; 16:225-9

21. Massler $M$. The P-M-A index for the assessment of gingivitis. J Periodontol 1967; 38 (Suppl):592-601.

22. Greene JC, Vermillion JR. The Oral Hygiene Index. A method for classifying oral hygiene status. J Am Dent Assoc 1960; 61:172-9

23. Loesche WJ, Lopatin DE, Giordano J, Alcoforado $G$, Hujoel P. Comparison of the benzoyl-DL-arginine-naphthylamide (BANA) test, DNA probes, and immunological reagents for ability to detect anaerobic periodontal infections due to Porphyromonas gingivalis, Treponema denticola, and Bacteroides forsythus. J Clin Microbiol 1992; 30:427-433.

24. Bretz WA, Eklund SA, Radicchi R, Schork MA, Schork N, Schottenfeld D, et al. The use of a rapid enzymatic assay in the field for the detection of infections associated with adult periodontitis. J Public Health Dent 1993; 53:235-40.
25. Wilson M, Lopatin D, Osborne G, Kieser JB. Prevalence of Treponema denticola and Porphyromonas gingivalis in plaque from periodontally healthy and periodontally diseased sites. J Med Microbiol 1993; 38:406-10

26. Halinen S, Sorsa T, Ding Y, Ingman T, Salo $\mathrm{T}$, Konttinen $\mathrm{YT}_{1}$ et al. Characterization of matrix metalloproteinase (MMP-8 and -9) activities in the saliva and in gingival crevicular fluid of children with Down's syndrome. J Periodontol 1996; 67:748-54.

27. Shapira J, Stabholz A. A comprehensive 30-month preventive dental health program in a preadolescent population with Down's syndrome: a longitudinal study. Spec Care Dent 1996; 16:33-37.

28. Randell DM, Harth S, Seow WK. Preventive dental health practices of non-institutionalized Down's syndrome children: a controlled study. J Clin Pediatr Dent 1992; 16:225-9.

29. Lopez-perez R, Broges-Yanez SA, JimenezGarcia G, Maupome G. Oral hygiene, gingivitis, and periodontitis in persons with Down syndrome. Spec Care Dentist 2002; 22:214-20.

30. Modeer T, Barr M, Dahllof G. Periodontal disease in children with Down's syndrome. Scand J Dent Res 1990; 98:228-34.

31. Reuland-Bosma W, Liem RS, Jansen HW, van-Dijk LJ, van-der-Weele LT. Morphological aspects of the gingiva in children with Down's syndrome during experimental gingivitis. J Clin Periodontol 1988; 15:293-302.

32. Nakagawa S, Fujii H, Machida Y, Okuda K. A longitudinal study from prepuberty to puberty of gingivitis. Correlation between the occurrence of Prevotella intermedia and sex hormones. J Clin Periodontol 1994; 21:658-65.

33. Nakagawa S, Machida Y, Nakagawa T, Fujii $H$, Yamada S, Takazoe I, et al. Infection by Porphyromonas gingivalis and

Actinobacillus actinomycetemcomitans, and antibody responses in different ages in humans. J periodontal Res 1994; 29:9-16.

34. Abraham J, Stiles HM, Kammerman LA, Forrester D. Assessing periodontal pathogens in children with varying levels of oral hygiene. J Dent child 1990; 57:189-93.

35. Robert JC, Gagnot G, Mouton C. Bacterial periodontal plaque in childhood. Literature review. J Parodontol 1991; 10:77-91.
36. Barr-Agholme $M$, Dahllof $G$, Linder $L$, Modeer T. Actinobacillus actinomycetemcomitans, Capnocytophaga and Porphyromonas gingivalis in subgingival plaque of adolescents with Down's syndrome. Oral Microbial Immunol 1992; 7:224-48.

37. Santos R, Shanfeld J, Casamassimo P. Serum antibody response to Actinobacillus actinomycetemcomitans in Down's syndrome. Spec Care Dent 1996; 16:80-3. 